\title{
Experimental Study of High-Range-Resolution Medical Acoustic Imaging for Multiple Target Detection by Frequency Domain Interferometry
}

\author{
Tomoki Kimura, Hirofumi Taki, Takuya Sakamoto, and Toru Sato \\ Graduate School of Informatics, Kyoto University, Yoshida-honmachi, Sakyo-ku, Kyoto 606-8501, Japan
}

Received November 19, 2008; accepted March 9, 2009; published online July 21, 2009

We employed frequency domain interferometry (FDI) for use as a medical acoustic imager to detect multiple targets with high range resolution. The phase of each frequency component of an echo varies with the frequency, and target intervals can be estimated from the phase variance. This processing technique is generally used in radar imaging. When the interference within a range gate is coherent, the cross correlation between the desired signal and the coherent interference signal is nonzero. The Capon method works under the guiding principle that output power minimization cancels the desired signal with a coherent interference signal. Therefore, we utilize frequency averaging to suppress the correlation of the coherent interference. The results of computational simulations using a pseudoecho signal show that the Capon method with adaptive frequency averaging (AFA) provides a higher range resolution than a conventional method. These techniques were experimentally investigated and we confirmed the effectiveness of the proposed method of processing by FDI. (C) 2009 The Japan Society of Applied Physics

\section{Introduction}

Medical acoustic imagers with high range resolution are highly desirable for diagnosing carotid artery atherosclerosis. Several methods have been proposed for improving the range resolution; ${ }^{1-4)}$ however, that is still a technically challenging problem. Kudo et al. $^{5)}$ reported another imaging technique: using the frequency spectrum of an rf echo signal to measure the wall thickness of the carotid artery. The theory is based on the change in the spectrum caused by the target interval. The technique allows only two targets to exist in the range of imaging. Since the 1960s adaptive beamforming techniques have been employed to reduce the effect of noise and the contribution of the undesired signal, resulting in high-resolution imaging. In 1969 Capon $^{6}$ proposed an adaptive beamforming method for radio astronomy. Shan and Kailath ${ }^{7)}$ introduced a spatial averaging technique into the Capon method to suppress the coherent interference. Mann and Walker ${ }^{8)}$ employed the Capon method for experimental data obtained from a single point target and confirmed the improvement of spatial resolution. In medical acoustic imaging, Sasso and Bacrie ${ }^{9)}$ investigated the improvement of spatial resolution experimentally using the Capon method with spatial averaging. In this paper, we apply the Capon method with spatial averaging techniques to frequency domain interferometry (FDI), which has been developed for radar imaging, to create a high range resolution medical acoustic imager. The proposed method can estimate the positions of multiple targets within a range gate. This method is suitable for medical acoustic imaging requiring high range resolution, such as carotid artery wall imaging.

\section{High-Resolution Imaging by FDI}

\subsection{The processing method of FDI}

In our previous work we estimated the interval between multiple targets within a range gate using the phases of signals at different frequencies. ${ }^{10)}$ The phase difference between two signals from different targets is proportional to the product of the frequency and the target interval. Thus, the phase difference varies with the frequency of the signal, as depicted in Fig. 1. The variance of the phase difference between two signals at different frequencies is proportional to the product of the frequency difference and the target interval. Therefore, we can estimate the target interval by utilizing the variance of the phase difference.

To obtain a range profile, we calculate the correlation values between signals at different frequencies. The signals are similarly expressed in a vertical form as follows. The output of an imager $y$ is given by

$$
\begin{aligned}
y & =\mathbf{X}^{\mathrm{T}} \mathbf{W}^{*}=\mathbf{W}^{\dagger} \mathbf{X}, \\
\mathbf{X} & =\left[\begin{array}{llll}
X_{1} & X_{2} & \cdots & X_{N}
\end{array}\right]^{\mathrm{T}}, \\
\mathbf{W} & =\left[\begin{array}{ll}
\mathbf{W}_{1}, \ldots, \mathbf{W}_{N}
\end{array}\right]^{\mathrm{T}},
\end{aligned}
$$

where $\mathbf{X}$ is a signal expressed by a set of frequency components in a vertical form, $\mathbf{W}$ is a weighting function, and $N$ is the number of frequency component samples of the signal. The output power $P$ is given by

$$
\begin{aligned}
P & =E\left[y y^{*}\right]=\mathbf{W}^{\dagger} \mathbf{R W}, \\
\mathbf{R} & =E\left[\mathbf{X X}^{\dagger}\right],
\end{aligned}
$$

where $E[\cdot]$ denotes the expectation and $\mathbf{R}$ is the correlation matrix of the input signals at each frequency. We then integrate the correlation values with the weighting function for phase correction.

\subsection{Capon method}

The beamformer method scans all distances within a range gate. When multiple targets exist within a range gate, the resolution of the beamformer method deteriorates because of the interference by targets at other positions. Therefore, we used the Capon method which minimizes the contribution from other distances subject to a constant response at a desired distance. ${ }^{6)}$ This problem is expressed as follows: ${ }^{11)}$

$$
\begin{aligned}
& \min P(r) \quad \text { subject to } \mathbf{C}^{\dagger} \mathbf{W}=1, \\
& \mathbf{C}=\left[e^{\mathrm{j} k_{1} r}, \ldots, e^{\mathrm{j} k_{N} r}\right]^{\mathrm{T}},
\end{aligned}
$$

where $r$ is the distance, $k_{j}$ is the wavenumber for frequency component $j$, and $\mathbf{C}$ is the constraint vector. This problem can be solved by Lagrange multiplier methods. The solution to eqs. (6) and (7) is given by

$$
P_{\text {cap }}(r)=\frac{1}{\mathbf{C}^{\dagger} \mathbf{R}^{-1} \mathbf{C}} .
$$

where $P_{\text {cap }}(r)$ is called the Capon range profile. 


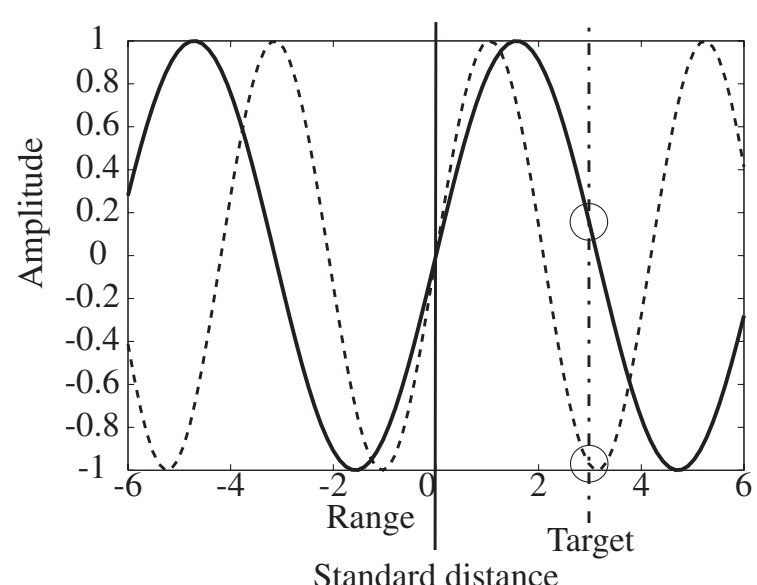

Fig. 1. Schema of the phases of the signals with different frequencies.

This method can estimate multiple targets within a range gate when the number of targets is less than the dimension of $\mathbf{R} .^{12)}$

\subsection{Suppressing the coherent interference by frequency averaging}

Since the time taken to obtain the data of a single range gate is sufficiently short, we assumed that the target intervals were constant while receiving the echo from a range gate. When an echo from a single range gate contains multiple coherent interferences, the cross correlation between the signal from the desired distance and the coherent interference is nonzero. The Capon method selects the weighting function that minimizes the sum of signals, thus resulting in the cancellation of the signal from the desired distance by the coherent interference. To solve this problem, we suppressed the correlation between the desired signal and coherent interferences by a frequency-averaging technique. The phase relation among the signals changes with their respective target distances. Thus, averaging the correlation at several frequencies can suppress the correlation between a desired signal and coherent interferences, i.e., frequency averaging uses the same principle as spatial averaging. ${ }^{12)}$

We defined a correlation matrix of the $n$th subarray $\mathbf{R}_{n}$ and $K$-element subarrays to employ a frequency-averaging technique, as shown in Fig. 2. The frequency-averaged correlation matrix $\mathbf{R}^{\prime}$ is given by

$$
\mathbf{R}^{\prime}=\sum_{n=1}^{N} v_{n} \mathbf{R}_{n},
$$

where $v_{n}$ are averaging weights for the correlation matrices of the subarrays. They are real and subject to the following equation:

$$
\sum_{n=1}^{N} v_{n}=1 .
$$

Two averaging techniques have been proposed to suppress the correlation. ${ }^{13)}$ Uniform frequency averaging (UFA) averages the correlation in uniform weights. In this case, averaging weights are defined as follows:

$$
v_{n}=\frac{1}{N}, \quad(n=1, \ldots, N) .
$$

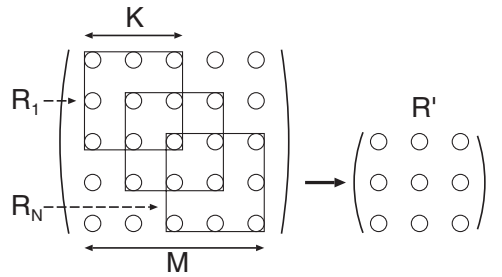

Fig. 2. Relationship between the correlation matrices of the full array and each subarray.

Adaptive frequency averaging (AFA) averages the correlation utilizing controlled weights to suppress the correlation completely. When the correlation is suppressed completely, the correlation matrix $\mathbf{R}^{\prime}$ becomes a Toeplitz matrix, which has equal elements along each diagonal. Thus, we set the averaging weights so as to equalize the elements in the diagonal of the averaged matrix $\mathbf{R}^{\prime}$. A measure of the deviation of the correlation matrix for a Toeplitz matrix is given by

$$
\begin{aligned}
& \varepsilon=\sum_{i=0}^{K-2} \sum_{k=1}^{K-i}\left|r_{k+i, k}{ }^{\prime}-r^{\prime}(i)\right|^{2} \\
& r^{\prime}(i)=\frac{1}{K-i} \sum_{k=1}^{K-i} r_{k+i, k}{ }^{\prime}=\frac{1}{K-i} \sum_{k=1}^{K-i}\left(\sum_{n=1}^{N} v_{n} r_{k+i, k \cdot n}\right) \\
& (i=0, \ldots, K-2)
\end{aligned}
$$

where $r_{k+i, k \cdot n}$ is the $(k+i, k)$ element of $\mathbf{R}_{n}$. Equation (12) can be also rewritten as follows:

$$
\begin{gathered}
\varepsilon=\mathbf{V}^{\mathrm{T}} \mathbf{R}_{\mathrm{ee}} \mathbf{V} \\
V=\left[v_{1}, v_{2}, \ldots, v_{N}\right]^{\mathrm{T}} \\
R_{e e}=\sum_{i=0}^{K-2} \sum_{k=1}^{K-i} \operatorname{Re}\left\{\mathbf{e}_{k+i, k} \cdot \mathbf{e}_{k+i, k}^{\dagger}\right\} \\
\mathbf{e}_{k+i, k}=\left[e_{k+i, k \cdot 1}, \ldots, e_{k+i, k \cdot n}\right]^{\mathrm{T}} \\
e_{k+i, k \cdot n}=r_{k+i, k \cdot n}-\frac{1}{K-i} \sum_{k=1}^{K-i} r_{k+i, k \cdot n}, \\
(i=0, \ldots, K-2 ; k=1, \ldots, K-i ; n=1, \ldots, N)
\end{gathered}
$$

where $\operatorname{Re}\{\cdot\}$ denotes the real part and $e_{k+i, k \cdot n}$ is the deviation of the $(k+i, k)$ element of $\mathbf{R}_{n}$ from the mean value along its $i$ th subdiagonal. Therefore, this problem can be expressed as follows:

$$
\min \left(\varepsilon=\mathbf{V}^{\mathrm{T}} \mathbf{R}_{\mathrm{ee}} \mathbf{V}\right) \quad \text { subject to } \mathbf{V}^{\mathrm{T}} \mathbf{I}=1,
$$

where I denotes an $\mathrm{N}$-dimensional vector in which all the elements are unity. The optimum averaging weights is

$$
\mathbf{V}_{\text {opt }}=\mathbf{R}_{\mathrm{ee}}{ }^{-1} \mathbf{I}\left(\mathbf{I}^{\mathrm{T}} \mathbf{R}_{\mathrm{ee}}{ }^{-1} \mathbf{I}\right)^{-1} \text {. }
$$

The resolution of AFA is generally higher than that of UFA. ${ }^{12)}$ In this case, this method can estimate multiple targets within a range gate when the number of targets is less than the dimension of $\mathbf{R}^{\prime}{ }^{12)}$

\subsection{Whitening}

The capon method assumes that a narrowband signal is utilized, ${ }^{14,15)}$ i.e., the transmit powers of sampled frequency components of the signal are equal. When we utilize a wideband signal for acoustic imaging, the transmit power 


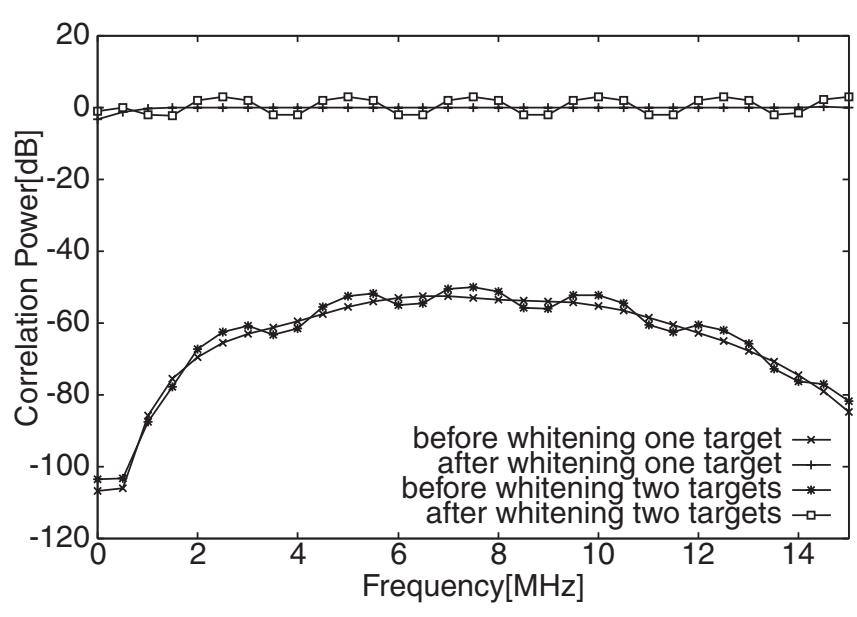

Fig. 3. Whitening process for the spectrum of the transmitted signal after applying a matched filter.

varies with its frequency. To solve this problem we corrected the transmit power of all sampled frequency components uniformly. A frequency component of a received signal is expressed by the following equation:

$$
\begin{aligned}
X_{l}(\omega) & =F(\omega) G^{*}(\omega) \\
& =|G(\omega)|^{2}\left\{k_{1}(\omega) e^{-\mathrm{j} \omega \tau_{1}}+k_{2}(\omega) e^{-\mathrm{j} \omega \tau_{2}}\right\},
\end{aligned}
$$

where $\omega$ is the angular frequency of the $l$ th frequency component of the received signal, $F(\omega)$ is the echo spectrum, $G(\omega)$ is the reference spectrum, $k_{i}(\omega)$ is a parameter of the waveform change due to the $i$ th target, and $\tau_{i}$ is the delay time for the $i$ th target. In this study we assumed that the waveform of echoes is the same as that of the reference wave. Therefore, the whitening correlation spectrum $X_{\text {whil }}(\omega)$ is given by

$$
\begin{aligned}
X_{\mathrm{whi} l}(\omega) & =\frac{1}{|G(\omega)|^{2}+\eta} X_{l}(\omega) \\
& =\frac{|G(\omega)|^{2}}{|G(\omega)|^{2}+\eta}\left\{a e^{-\mathrm{j} \omega \tau_{1}}+b e^{-\mathrm{j} \omega \tau_{2}}\right\},
\end{aligned}
$$

where $\eta$ is the noise power of the received signal, $a$ and $b$ are constants, and $a / b$ is the amplitude ratio of the two backscattered waves. $\eta$ can be estimated from the variance of the power spectrum of a received signal when it is uniform in a certain frequency band. However, the noise estimation method is not adequate for the case of using a wideband signal with variation of a power spectrum. By assuming the noise to be white, we estimated the power density of the noise with a received signal in a higherfrequency band, where the signal power is negligible. In this paper, a frequency band of 6.25 to 56.25 for the center frequency is empirically chosen for the noise power estimation. Figure 3 shows the whitening correlation spectrum of the echo from an acrylic board. The time interval between the two targets is $0.4 \mu \mathrm{s}$.

\section{Numerical Experiment}

We evaluated the effectiveness of the proposed technique by a computational simulation using a pseudoecho. We assumed that two boundaries existed $0.05 \mathrm{~mm}$ apart and that an echo consisted of two backscatters from the two boundaries. We applied the pseudoecho

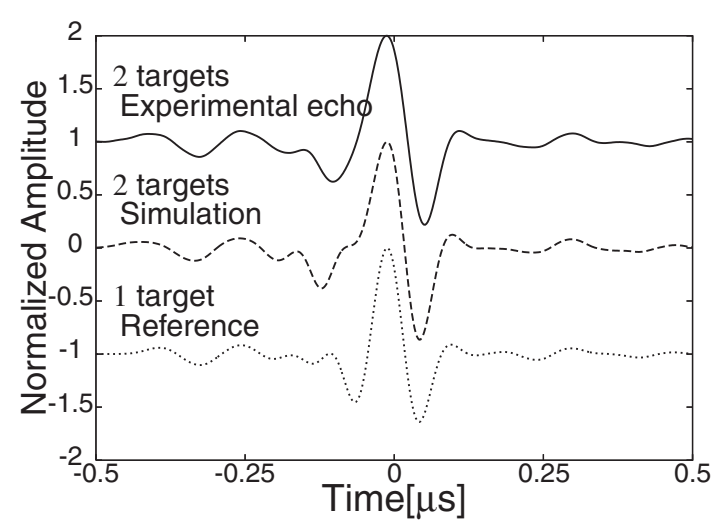

Fig. 4. Echo from a $0.05-\mathrm{mm}$-thick polyethylene sheet, the pseudoecho $s_{1}(t)$, and the echo from an acrylic board.

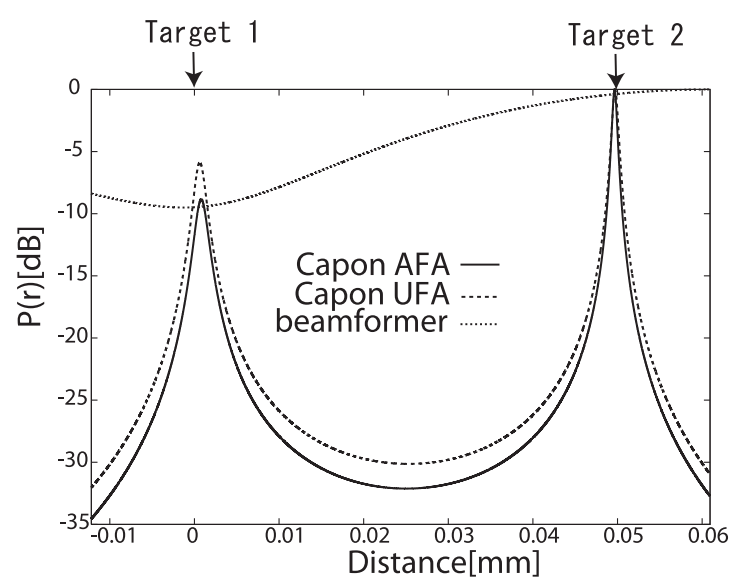

Fig. 5. Normalized brightness distribution from a pseudoecho where the two targets are located at a distance of $0.05 \mathrm{~mm}$. Target distances are estimated using the beamformer method, the Capon method with UFA, and AFA.

$$
s_{1}(t)=a s_{0}(t)+b s_{0}(t-\tau)+n(t),
$$

where $s_{0}(t)$ is the echo from an acrylic board surface, as shown in Fig. 4, $\tau$ is the time delay resulting from the thickness of the sheet, and $n(t)$ is white noise. The parameters of the pseudoecho shown in Fig. 4 are $a / b=$ $0.493, \tau=0.051 \mu \mathrm{s}$, and $\mathrm{S} / \mathrm{N}=38.3 \mathrm{~dB}$. We used the echo from the acrylic board surface as a reference wave. Figure 5 shows the normalized brightness distribution of the pseudoecho given by the beamformer method, the Capon method with UFA, and the Capon method with AFA. In this study, we employed 26 equally spaced frequencies within the range of 1.5 to $14.0 \mathrm{MHz}$, where these frequencies have a power of $-20 \mathrm{~dB}$ above the maximum power. The beamformer method hardly detected the two signals from the echo. Utilizing the Capon method, the two edges were detected clearly and the half-power widths of the two edges were 1.55 and $0.77 \mu \mathrm{m}$ for UFA and 1.69 and $0.62 \mu \mathrm{m}$ for AFA. The error in the estimated thickness was $0.92 \mu \mathrm{m}$ for UFA and $1.15 \mu \mathrm{m}$ for AFA. In the ideal case without noise, we can estimate the echo power precisely.

\section{Experimental Study}

In this study we also investigated the proposed technique experimentally. Figure 6 shows a schema of the experiment. The target was a $0.05-\mathrm{mm}$-thick polyethylene sheet. The 


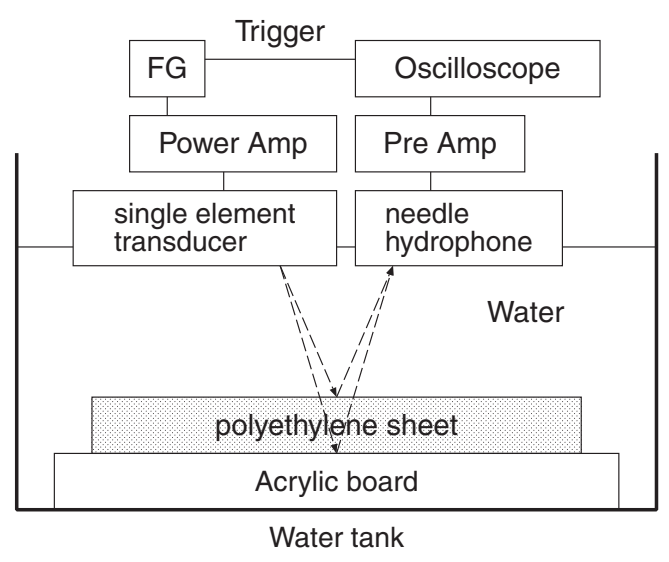

Fig. 6. Schema of the experimental setup.

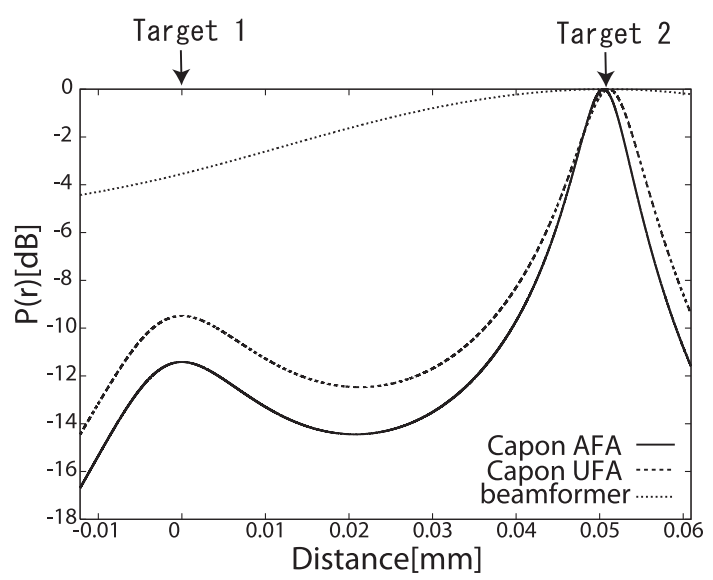

Fig. 7. Normalized brightness distribution of an echo from a polyethylene sheet. (The parameters are the same as in Fig. 4.)

transmit waveform was a monocycle pulse, the center frequency was $8 \mathrm{MHz}$, and the sampling frequency was $500 \mathrm{MHz}$. The echo consisted of two backscattered waves from the front and back of the polyethylene sheet. We detected the two waves from the echo using FDI with the Capon method and thus estimated the thickness of the sheet.

In this study we employed the echo from a polyethylene sheet with the coherent integration of 10000 pulses, as shown in Fig. 4. The noise power of this echo with coherent integration is $38.3 \mathrm{~dB}$, where the frequency band utilized for noise power estimation is 50 to $450 \mathrm{MHz}$. Figure 7 shows the normalized brightness distribution of this signal. In this case, we also used the echo from an acrylic board surface as a reference wave and the same parameters for imaging as those used in the computational testing. It was difficult to separate the echo employing the beamformer method. Utilizing the Capon method, the two edges were detected and the error in estimated thickness was $1.08 \mu \mathrm{m}$ for UFA and $0.46 \mu \mathrm{m}$ for AFA.

\section{Conclusions}

In this paper, we proposed a medical acoustic imaging technique using FDI with the Capon method. The results of computational testing indicate that the beamformer method does not distinguish between two target boundaries that lie $0.05 \mathrm{~mm}$ apart and that the Capon method with AFA provides a better range resolution than the Capon method with UFA. Utilizing the Capon method, the half-power widths of the two edges were 1.55 and $0.77 \mu \mathrm{m}$ for UFA and 1.69 and $0.62 \mu \mathrm{m}$ for AFA. The error in estimated thickness was $0.92 \mu \mathrm{m}$ for UFA and $1.15 \mu \mathrm{m}$ for AFA. The results of the experimental study showed that the beamformer method has the worst performance because of its poor suppression of correlation resulting from coherent interference. Two signals were detected from the echo from a polyethylene sheet by the Capon method with UFA and AFA. The error in estimated thickness using the Capon method was $1.08 \mu \mathrm{m}$ for UFA and $0.46 \mu \mathrm{m}$ for AFA. The range resolution in the experimental study deteriorates, especially at the range of the first boundary. One of the reasons for this may be that in calculating the frequency spectrum, we approximate the auto correlation function of the echo to the cross-correlation function of the echo and the reference signal. The echo from an acrylic board surface, the reference signal used in this study, is different from the first echo from a polyethylene sheet surface. The optimization of the reference wave to improve the range resolution of the proposed method will be a future task.

1) N. Nakagawa, H. Hasegawa, and H. Kanai: Jpn. J. Appl. Phys. 43 (2004) 3220.

2) C. Arihara, H. Hasegawa, and H. Kanai: Jpn. J. Appl. Phys. 45 (2006) 4727.

3) J. Inagaki, H. Hasegawa, H. Kanai, M. Ichiki, and F. Tezuka: Jpn. J. Appl. Phys. 45 (2006) 4732.

4) K. Kudo, H. Hasegawa, and H. Kanai: Jpn. J. Appl. Phys. 46 (2007) 4873.

5) N. Kudo, X. Zhang, and K. Yamamoto: J Med. Ultrason. 25 (1998) 155.

6) J. Capon: Proc. IEEE 57 (1969) 1408.

7) T. J. Shan and T. Kailath: IEEE Trans. Acoust. Speech Signal Process. 33 (1985) 527.

8) J. A. Mann and W. F. Walker: Proc. IEEE Ultrasonics Symp., 2002, p. 1807.

9) M. Sasso and C. C. Bacrie: Proc. IEEE, ICASSP, 2005, p. 489.

10) H. Luce, M. Yamamoto, S. Fukao, D. Helal, and M. Crochet: J. Atmos. Terr. Phys. 63 (2001) 221.

11) L. Smaini, H. Luce, M. Crochet, and S. Fukao: J. Atmos. Oceanic Technol. 19 (2001) 954.

12) K. Takao and N. Kikuma: IEEE Trans. Antennas Propag. 35 (1987) 1389.

13) O. L. Frost: IEEE ICASSP, 1986, p. 2499.

14) B. D. Van Veen and K. M. Buckley: IEEE ASSP Mag. 5 (1988) No. 2, 4.

15) O. L. Frost, III: Proc. IEEE 60 (1972) 926. 